\title{
FTIR and Raman analyses of the Tagish Lake meteorite: Relationship with the aliphatic hydrocarbons observed in the Diffuse Interstellar Medium
}

\author{
G. Matrajt ${ }^{1}$, J. Borg ${ }^{1}$, P. I. Raynal ${ }^{1}$, Z. Djouadi ${ }^{1}$, L. d'Hendecourt ${ }^{1}$, G. Flynn ${ }^{2}$, and D. Deboffle ${ }^{1}$ \\ 1 IAS, Bât. 121, Université Paris-Sud, 91405 Orsay Cedex, France \\ 2 Department of Physics, SUNY-Plattsburgh, Plattsburgh NY, 12901, USA
}

Received 16 October 2003 / Accepted 27 November 2003

\begin{abstract}
Using FTIR and Raman microspectroscopies we have analysed 6 fragments of the Tagish Lake meteorite. The data obtained show that all the fragments belong to the carbonate-rich lithology, where an organic material, including a highly disordered macromolecular carbonaceous component is found. The FTIR approach shows that part of the organic material present in Tagish Lake is aliphatic. The Raman approach shows that there is also highly disordered polyaromatic organic material, which is abundant. Furthermore, the comparison of Raman data of Tagish Lake to other carbonaceous chondrites (CI, CM2, CR2) shows that the carbon in Tagish Lake is different, supporting the assertion that this meteorite is a unique and new type of carbonaceous chondrite. The comparison of the aliphatic hydrocarbon FTIR data found in the Tagish Lake meteorite with the aliphatic hydrocarbon IR data of the carbonaceous chondrites Orgueil and Murchison and with the diffuse Interstellar Medium (ISM) shows that they are different, in that the Tagish Lake meteorite has longer aliphatic chains.
\end{abstract}

Key words. meteors, meteoroids - techniques: spectroscopic - methods: analytical - ISM: molecules - astrochemistry

\section{Introduction}

The Tagish Lake Meteorite fell in Yukon, Canada on January 2000 on the frozen surface of the Taku Arm branch of Tagish Lake (Brown et al. 2000). It is a carbonaceous chondrite classified as CI2 according to its mineralogy (Brown et al. 2000; Zolensky et al. 2002). On the basis of reflectance spectral data, its parent body is believed to be related to the D-type asteroids (Hiroi et al. 2001). Tagish Lake has been studied with several techniques for its mineralogy (Zolensky et al. 2002; Nakamura et al. 2003b), its light elements content (Grady et al. 2002), its organic chemistry (Pizzarello \& Huang 2002; Kminek et al. 2002; Cody et al. 2002; Nakamura et al. 2002, 2003a), its trace elements content (Friedrich et al. 2002), its geochemistry (Baker et al. 2002; Mittlefehldt 2002; Leshin et al. 2001), and its noble gases (Grady et al. 2001).

FTIR (Fourier Transform Infra Red) and Raman microspectroscopies present the advantage of being nondestructive techniques, if we exclude the facts that i) for FTIR, the grains are crushed on an infrared transparent window (Raynal et al. 2000) and ii) for Raman studies, the power of the incident laser needs to be low enough to avoid heating of the sample. Therefore, these analytical techniques are highly appreciated for analysing precious samples such as meteorites and IDPs. Furthermore, the infrared and Raman

Send offprint requests to: G. Matrajt, e-mail: graciela.matrajt@ias.u-psud.fr spectra of a given sample usually differ because IR activity requires a change in dipole moment during the transition whereas Raman activity requires a change in polarizability, hence each technique can provide complementary information regarding the sample. With these two techniques, both the carbonaceous components and the mineralogy of the matrix can be investigated with a high spatial resolution (a few microns), allowing the detailed analysis of heterogeneous samples.

To date, only few studies with FTIR exist on Tagish Lake (Flynn et al. 2001; Keller \& Flynn 2001; Nakamura et al. 2002, 2003a). Flynn et al. (2001) and Keller \& Flynn (2001) have studied pieces of Tagish Lake mainly composed of a carbonate-rich lithology. During their studies, the $\mathrm{Si}-\mathrm{O}$ stretching from the phyllosilicates (at $1017 \mathrm{~cm}^{-1}$ ), the carbonate feature (at $1424 \mathrm{~cm}^{-1}$ ) and the organic aliphatic $\mathrm{C}-\mathrm{H}$ stretching (in the region 2700-3000 $\mathrm{cm}^{-1}$ ) were observed. Two weak absorption features were also observed at 2988 and $2995 \mathrm{~cm}^{-1}$, not seen before in carbonaceous chondrites such as Orgueil or Murchison. On the other hand, Nakamura et al. (2002, 2003a) studied several pieces of the carbonate-poor lithology of Tagish Lake. In particular, they analysed in situ the matrix and amorphous carbon globules (called hollow organic globules). During their studies, the lattice vibration from phyllosilicates and the sharp $\mathrm{C}-\mathrm{H}$ aliphatic vibrations were observed. Furthermore, bands corresponding to aromatics (in the region $1500-1600 \mathrm{~cm}^{-1}$ ), together with oxygenated functions related to esters (at 1280 and $1740 \mathrm{~cm}^{-1}$ ) were also observed. 
Several studies using Raman spectroscopy have been performed on various carbonaceous chondrites (Christophe Michel-Lévy \& Lautié 1981; Wopenka \& Sandford 1984; Heymann et al. 1986; Makjanic et al. 1989; Macklin et al. 1990; Rietmeijer 1990) and interplanetary dust particles (Wopenka 1988). However, only one Raman study exists to date on Tagish Lake (Nakamura et al. 2002). In this latter work, only organic-rich pieces of the carbonatepoor lithology of the meteorite were analysed with Raman spectroscopy.

To evaluate if the differences observed with FTIR on both lithologies are also observed with Raman spectroscopy, in this paper we analyse several randomly chosen fragments of the carbonate-rich lithology of Tagish Lake, using both FTIR and Raman microspectroscopies. We compare the Raman results obtained on Tagish Lake with previous Raman studies made on several CI, CR, and CM carbonaceous chondrites (Raynal 2003), to explore their relationship. We also evaluate if the Raman approach confirms the CI2 classification attributed to Tagish Lake using other analytical techniques such as Transmission Electron Microscopy [TEM] (Nakamura et al. 2003b), Stepped Combustion (Grady et al. 2002), Induced Coupled Plasma Mass Spectrometry [ICPMS] (Friedrich et al. 2002), Gas Chromatography Mass Spectrometry [GCMS] (Pizzarello \& Huang 2002), stepped pyrolysis (Baker et al. 2002), and Secondary Ion Mass Spectrometry [SIMS] (Leshin et al. 2001). Finally we compare the IR spectra, particularly the aliphatic and aromatic features and the methylene $\left(\mathrm{CH}_{2}\right)$ to methyl $\left(\mathrm{CH}_{3}\right)$ ratio, to the ones currently observed in the diffuse Interstellar Medium (Sandford et al. 1991; Pendleton et al. 1994; Sandford et al. 1995), as the similarity between primitive organic materials in meteorites and interstellar organic matter has since long been postulated on the basis of such comparison (Ehrenfreund et al. 1991).

\section{Materials and methods}

The sample was recovered from the land adjacent to the lake several months after the fall, when the snow had melted (Flynn et al. 2001), and purchased from The Meteorite Market.

\subsection{Sample preparation}

All the sample preparation was performed in ultra-clean conditions, in a class 10000 clean room. The sample was carefully checked by optical microscopy to look for residues on its surface that could come from the environment (mud, leaves) where it was recovered or from the package (adhering materials, plastic bag) where it was shipped. Fortunately, the sample was very clean and there was no need to remove any of the exterior. However, to avoid surface contamination and the fusion crust we fragmented the $20 \mathrm{mg}$ sample and took only pieces coming from its interior. We took 6 pieces of a few tens of $\mu \mathrm{m}$ in size for the analyses. To diminish scattering processes and interference structures which produce spectral artifacts during the FTIR analysis, the samples were crushed using the procedure developed by Raynal et al. (2000). Five fragments (named TL1, 2, 3, 4, 5) were placed on freshly prepared $\mathrm{KBr}$ windows. A sixth fragment (TL6) was crushed on a pure CsI window purchased from MAT-TECHNOLOGY.

\subsection{Analytical techniques}

\subsubsection{FTIR}

We used Fourier Transform InfraRed spectroscopy, using a Nicolet Magna-IR 560 ESP spectrometer coupled to a Nicolet Nicplan infra-red microscope, in line with a Synchrotron Radiation Source located on line SA5 at the Laboratoire pour l'Utilisation du Rayonnement Electromagnétique (LURE) at the University of Paris-Sud, Orsay, France. The spectrometer is equipped with a $\mathrm{KBr}$ beamsplitter and a nitrogencooled Mercury-Cadmium-Tellure (MCT) detector working in the 4000 to $650 \mathrm{~cm}^{-1}(2.5$ to $15 \mu \mathrm{m})$ spectral range. The microscope uses a Schwarzchild-Cassegrain objective $(32 \times)$ and a condenser $(10 \times)$ in the transmission mode. Besides the Synchrotron light source, the standard light source of the instrument, a conventional black body-like source (Globar) is available. Further details about this instrument and the advantages of both light sources can be found in Raynal et al. (2000). Since no significant differences appeared between the spectra obtained with the Globar source and the Synchrotron source, most of the analyses described here were performed using the Globar source, more often available. To enhance the signal to noise ratio and minimize background fluctuations due to variations in the purge, 10 acquisitions of 256 scans each at the resolution of $4 \mathrm{~cm}^{-1}$ were made for each sample and then averaged.

\subsubsection{Raman}

We employed micro-Raman spectroscopy using a Labram HR 800 instrument coupled to a confocal microscope (Olympus DIXM-ILHS) at the Laboratoire des Sciences de la Terre, ENS Lyon, France. The excitation source was the 514.5-nm line of an Ar laser, used with a power kept at $20 \mu \mathrm{W}$, to avoid over-heating the sample. Two objectives $(50 \times$ and $100 \times)$ were used to focalise the spot. We used the spectrometer between 200 and $4000 \mathrm{~cm}^{-1}$. Acquisition times were kept between 30 and $60 \mathrm{~s}$. Two acquisitions per spot were made and co-added to improve the signal to noise ratio. The spurious effects (spikes) often found in Raman spectra (due to cosmic rays) were removed from the spectra. The samples presented an intense fluorescence signal which did not allow the detection of the mineral phases such as phyllosilicates and carbonates, so that only the two carbon bands (at 1378 and $1593 \mathrm{~cm}^{-1}$ ) were present. After a baseline correction, the Raman spectra were fitted by a two-band model, to extract the individual band parameters (position of maximum, full width at half maximum, peak intensity and band area), as described in Raynal (2003). We chose the two-Lorentzian band model for spectrum fitting to facilitate the comparison with Raman data of carbon obtained on other CI1, CM2 and CR2 carbonaceous chondrites (Raynal 2003). 


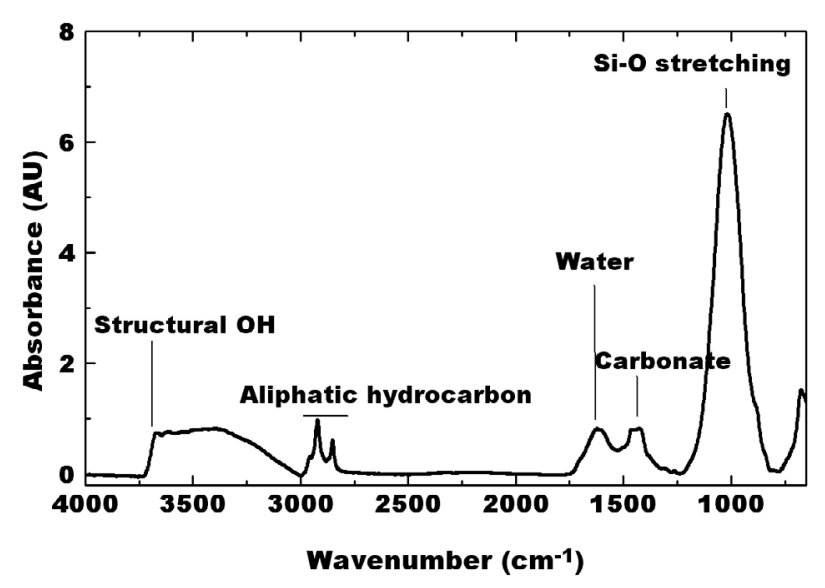

Fig. 1. A typical FTIR spectrum of Tagish Lake after a linear baseline correction. The saponite component is represented by the $\mathrm{Si}-\mathrm{O}$ stretching at $1017 \mathrm{~cm}^{-1}$, the water stretching at $1615 \mathrm{~cm}^{-1}$ and the structural $\mathrm{OH}$ stretching at $3668 \mathrm{~cm}^{-1}$. The carbonate component is represented by the broad band centered at $1450 \mathrm{~cm}^{-1}$. The aliphatic stretching component is represented by the group of peaks in the $3000-2700 \mathrm{~cm}^{-1}$ region.

\subsubsection{Characteristics of the FTIR and Raman microspectroscopies}

The samples used for this study have been crushed. This could be considered to be a destructive approach since the crushing probably alters the distribution of the mineral phases. However, comparing to chemical analytical techniques such as gas and liquid chromatographies or stepped combustion where the sample is totally lost, or techniques such as SIMS and ICPMS where samples are strongly corroded, the crushing approach presents the advantage of not chemically or physically destroying the sample. Therefore, both FTIR and Raman approaches can be considered non-destructive analytical tools. Furthermore, compared with other analytical techniques (TEM, Chromatography) the FTIR and Raman approaches present the advantage of being able to determine at a few microns scale, the nature of the carbon present in the sample, its distribution in the matrix and its relation to the different lithologies.

\section{Results}

\subsection{FTIR}

From Flynn's previous studies (Flynn et al. 2001; Keller \& Flynn 2001), this sample should originate from the carbonaterich lithology of Tagish Lake. However, since Tagish Lake is quite heterogeneous, we checked it by FTIR. Figure 1 shows a typical FTIR spectrum obtained with the TL6 sample.

The FTIR data reveal that the 6 fragments analysed during this study are mainly dominated by hydrated silicates and carbonates, confirming that this piece of the Tagish Lake meteorite belongs to the carbonate-rich lithology. Table 1 shows the peaks observed in the spectra and their possible interpretations, according to Salisbury et al. (1992) and Socrates (2001).

The hydrated silicate is represented by the absorption features at $1017 \mathrm{~cm}^{-1}$ (Si-O stretch), $3668 \mathrm{~cm}^{-1}$ (structural hydroxyl feature) and $1615 \mathrm{~cm}^{-1}$ (water in smectite), in agreement with a saponite identification according to TEM observations (Keller \& Flynn 2001). However, some bands have multiple interpretations. For instance, the carbonate is represented by a broad band centered at $1450 \mathrm{~cm}^{-1}$ showing small features at 1424, 1466 and $1503 \mathrm{~cm}^{-1}$ (see Fig. 2), but some of these peaks are also typical of organic compounds. In Fig. 3 we show an expanded spectrum of the region $679-880 \mathrm{~cm}^{-1}$. The weak peaks of this region can be interpreted either as being also absorption features from carbonates or as absorption features of aliphatics (see Table 1). But they can also be interpreted as being aromatic compounds, since this type of molecules have bands in this region, corresponding to the out-of plane $\mathrm{C}-\mathrm{H}$ bending modes. However, it is expected that these bands would be stronger than the aromatic modes found at higher frequencies, which does not seem to be the case in this sample (see Fig. 3). The presence of carbonate peaks in this sample is in agreement with the TEM identification of a mixture of siderite and magnesite (Keller \& Flynn 2001).

Three major peaks are found in the $2700-3000 \mathrm{~cm}^{-1}$ region, corresponding to aliphatic hydrocarbon stretching features. An expansion of this region is shown in Fig. 4, where a fourth, smaller peak at $2865 \mathrm{~cm}^{-1}$ can be distinguished. The pair of peaks at 2922 and $2851 \mathrm{~cm}^{-1}$ corresponds to the asymmetrical and symmetrical stretching vibrations of $\mathrm{CH}_{2}$ in an aliphatic hydrocarbon. The peaks at 2958 and $2865 \mathrm{~cm}^{-1}$ correspond to the asymmetrical and symmetrical stretching vibrations of $\mathrm{CH}_{3}$ also in an aliphatic hydrocarbon (D'Hendecourt \& Allamandola 1986; Flynn et al. 2001). The C-H bending vibrations of these aliphatic hydrocarbons are mainly represented by the peaks at $\sim 1466$ and $\sim 1379 \mathrm{~cm}^{-1}$ as well as small peaks in the region $1266-1353 \mathrm{~cm}^{-1}$, as shoulders on the carbonate peak (see Fig. 2). Aromatic C-H stretch features in the region around $3030 \mathrm{~cm}^{-1}$, organic ester-like peaks or the two weak absorptions at 2988 and $2995 \mathrm{~cm}^{-1}$ cited by Flynn et al. (2001) are not observed in the present analyses. However, there is one weak aromatic peak in the region $1500-1650 \mathrm{~cm}^{-1}$ (at $1624 \mathrm{~cm}^{-1}$ ) corresponding to the $\mathrm{C}=\mathrm{C}$ stretching feature, and several weak peaks in the region $650-900 \mathrm{~cm}^{-1}$ which also correspond to aromatics (out-of-plane $\mathrm{C}-\mathrm{H}$ bending deformations), suggesting that aromatics might be present. But the interferences with carbonate, with the water in interaction with the smectite, and the absence of peaks at $3030 \mathrm{~cm}^{-1}$, make this identification only tentative. A weak peak at $\sim 1700 \mathrm{~cm}^{-1}$ corresponding to the $\mathrm{C}=\mathrm{O}$ stretching vibration is present as a shoulder of the broad band centered near $1624 \mathrm{~cm}^{-1}$.

\subsection{Raman}

Raman analyses were performed on the same samples studied by FTIR. Figure 5 shows a typical Raman spectrum obtained for the TL2 sample, in the $500-2500 \mathrm{~cm}^{-1}$ Raman shift region. The values of the band parameters obtained from the fitting procedure and by averaging all the 6 samples are given in Table 2 .

We observe the two first order Raman bands of polyaromatic carbon materials, namely the D and G bands (Ferrari \& Robertson 2000). The D band is characteristic of a disordered carbon, whereas the $\mathrm{G}$ band is characteristic of an 
Table 1. Peaks observed with FTIR and their possible interpretation, after Salisbury et al. (1992) and Socrates (2001). Some bands have multiple interpretations.

\begin{tabular}{|c|c|c|}
\hline Peak value (in $\mathrm{cm}^{-1}$ ) & Feature type & Possible Interpretations \\
\hline 679 & & carbonate \\
\hline 740 & & carbonate/branched alkane \\
\hline 842 & & carbonate/straight chain alkane \\
\hline 880 & & carbonate/aliphatic \\
\hline $650-900$ & out-of-plane $\mathrm{CH}$ bending deformation & aromatics \\
\hline 1017 & $\mathrm{Si}-\mathrm{O}$ stretch & phyllosilicate \\
\hline 1266 & $\mathrm{CH}_{3}$ & aliphatic hydrocarbon \\
\hline 1315 & bending $\mathrm{CH}_{3}$ & aliphatic hydrocarbon \\
\hline 1353 & bending $\mathrm{CH}_{3}$ & aliphatic hydrocarbon \\
\hline 1379 & bending symmetric $\mathrm{CH}_{3}$ & aliphatic hydrocarbon \\
\hline 1424 & $\mathrm{C}=\mathrm{C}$ stretching & aromatic/carbonate \\
\hline 1466 & bending asymmetric $\mathrm{CH}_{3}$ & aliphatic/carbonate \\
\hline 1503 & & carbonate \\
\hline $1320-1530$ & broad band centered at $\sim 1450$ & carbonate \\
\hline 1602 and 1615 & H-O-H stretching of water & phyllosilicate \\
\hline 1624 & $\mathrm{C}=\mathrm{C}$ stretching & aromatic/alkene \\
\hline 1700 & $\mathrm{C}=\mathrm{O}$ stretching & saturated aliphatic ketone/ carboxylic acid \\
\hline 2851 & stretching symmetric $\mathrm{CH}_{2}$ & aliphatic hydrocarbon \\
\hline 2865 & stretching symmetric $\mathrm{CH}_{3}$ & aliphatic hydrocarbon \\
\hline 2922 & stretching asymmetric $\mathrm{CH}_{2}$ & aliphatic hydrocarbon \\
\hline 2958 & stretching asymmetric $\mathrm{CH}_{3}$ & aliphatic hydrocarbon \\
\hline $3623-3680$ & free $\mathrm{OH}$ & water \\
\hline 3668 & structural hydroxyl feature & phyllosilicate \\
\hline
\end{tabular}

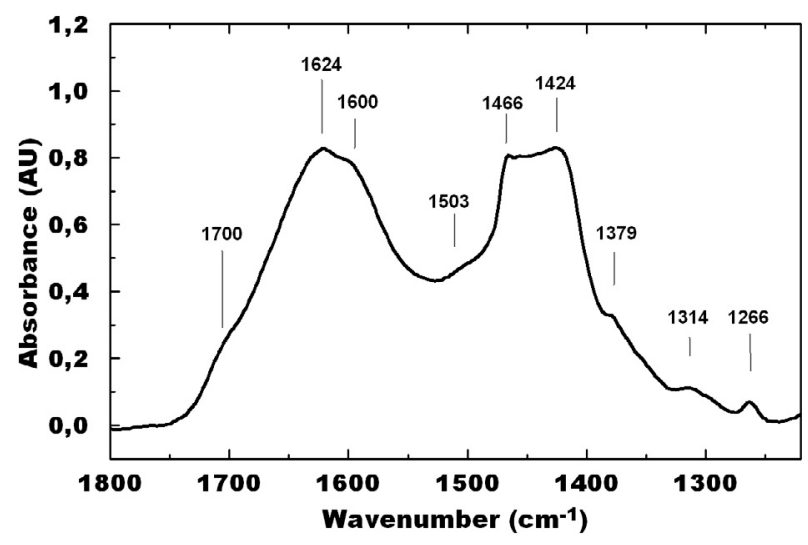

Fig. 2. The carbonate peaks and aliphatic hydrocarbon bending features in the $1250-1800 \mathrm{~cm}^{-1}$ region. The interpretation of the various peaks is given in Table 1 .

aromatic carbon (graphite type or polyaromatic solid). Wide D and $G$ bands such as the ones obtained here are characteristic of highly disordered carbonaceous materials. Furthermore, a significant fluorescence emission background in the Raman spectra (Fig. 5, top) is evidence of a structure with conjugated bonds (Raynal 2003). In order to compare the Tagish Lake carbon to the one found in other primitive carbonaceous chondrites (Orgueil, Alais, Ivuna [CI]; Murchison, Cold Bokkeveld, Murray [CM2]; Renazzo [CR2]), we have plotted the Raman

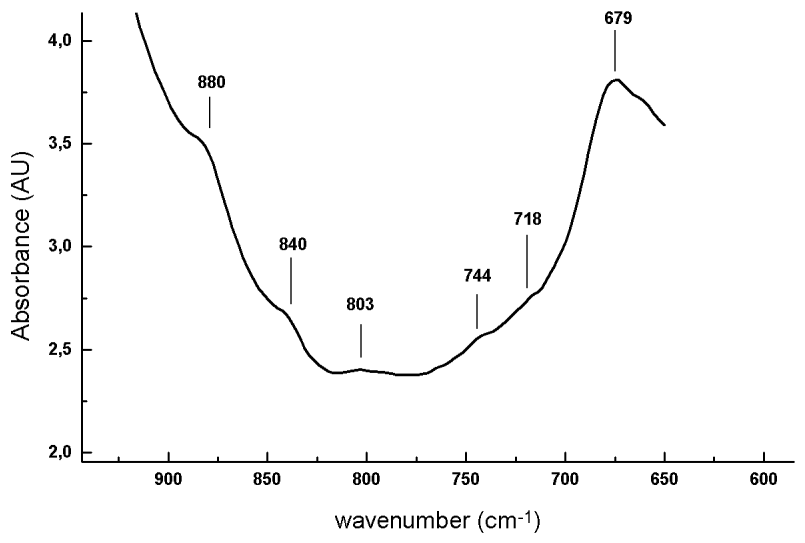

Fig. 3. The peaks of the $679-880 \mathrm{~cm}^{-1}$ region. Multiple interpretations exist for the various peaks, which are given in Table 1.

band characteristics in 3 diagrams (Fig. 6), according to a) the peak intensities ratio vs. the $\mathrm{D}$ band relative peak areas, b) the width vs. the position for the D band and c) the width vs. the position for the $\mathrm{G}$ band. The representative points of Tagish Lake fall in a region that extends the continuous distribution of the other primitive carbonaceous chondrites and Orgueil, with some points overlapping with members of the other groups. 


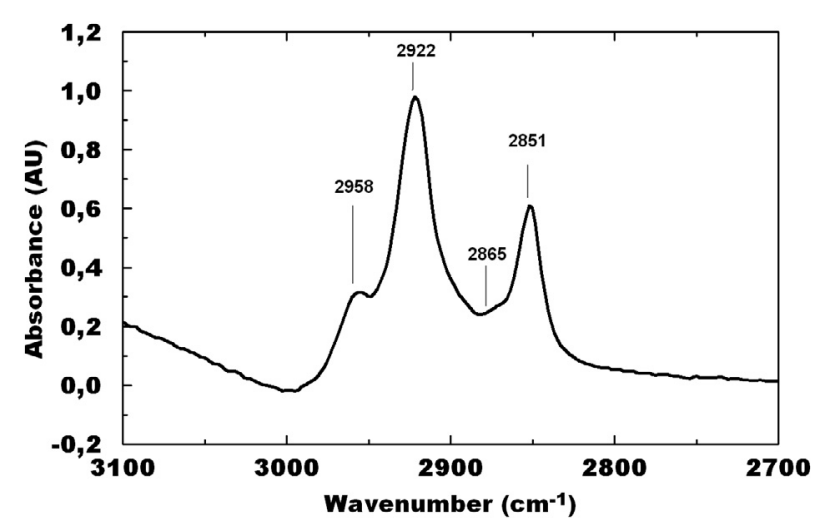

Fig. 4. The $\mathrm{C}-\mathrm{H}$ stretches of aliphatic hydrocarbons in the $2700-3100 \mathrm{~cm}^{-1}$ region. The interpretation of the various peaks is given in Table 1.
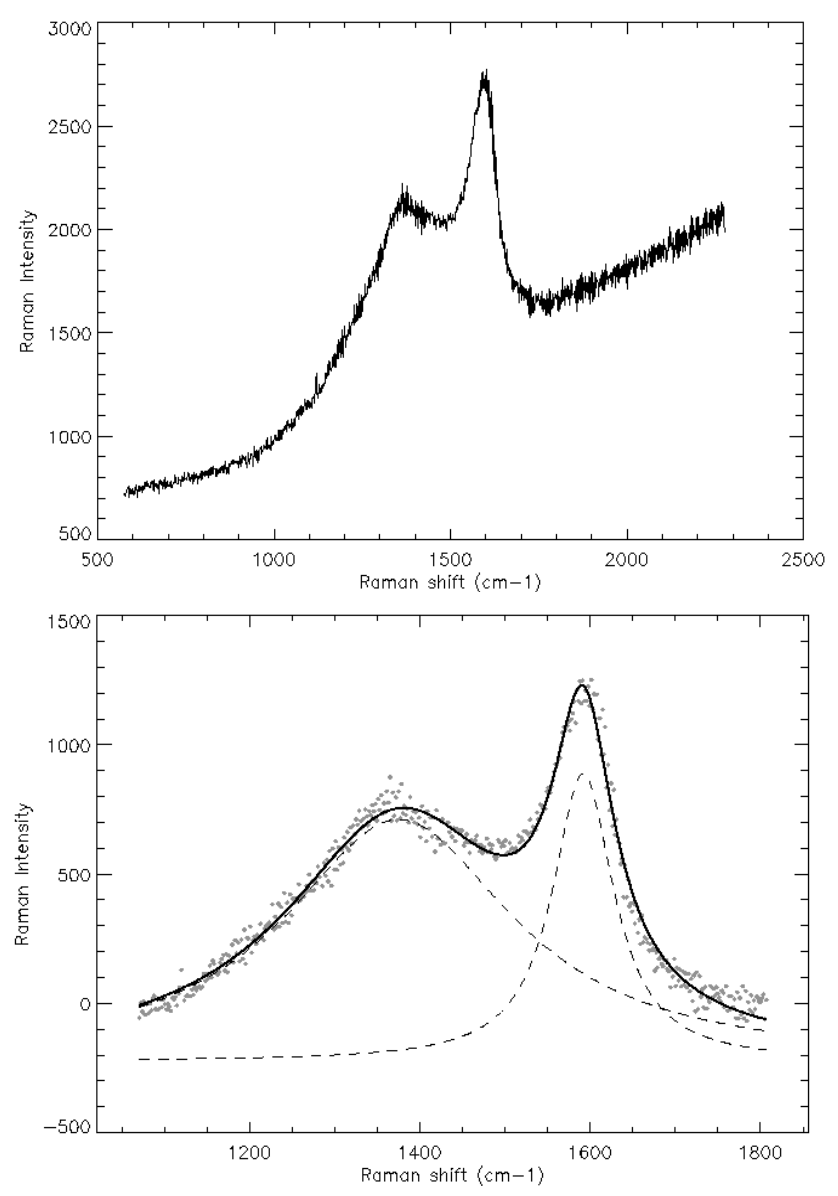

Fig. 5. A typical Raman spectrum of Tagish Lake. Top: the two first order Raman bands D (left) and G (right) caracteristic of highly disordered carbon. This spectrum also exhibits a significant fluorescence emission, as evidenced by the slope and the intensity of the background under the Raman bands. Bottom: the Raman spectrum fitted following a linear baseline correction and a two-Lorentzian band model.

\section{Discussion}

\subsection{FTIR}

The FTIR analyses do not reveal the presence of esters in our samples, which are carbonate-rich as previously stated. Instead,
Table 2. Raman parameters of the observed D and G bands of carbon. The values presented are an average of all the six samples analysed. Legend: $v=$ wavenumber, in $\mathrm{cm}^{-1} . I=$ intensity at the highest point of the peak. $A=$ area of the integrated peak, in $\% . F W H M=$ full width at half maximum, in $\mathrm{cm}^{-1}$.

\begin{tabular}{cc}
\hline \hline & 2-Lorentzian Model \\
\hline$v_{\max }(\mathrm{D})$ & $1378.80 \pm 7.27$ \\
$v_{\max }(\mathrm{G})$ & $1593.89 \pm 1.47$ \\
$F W H M(\mathrm{D})$ & $328.11 \pm 19.23$ \\
$F W H M(\mathrm{G})$ & $88.56 \pm 7.54$ \\
$I_{(\mathrm{D})} / I_{(\mathrm{G})}$ & $0.85 \pm 0.06$ \\
$A_{(\mathrm{D})} /\left(A_{(\mathrm{D})}+A_{(\mathrm{G})}\right)$ & $71.51 \pm 1.60$ \\
\hline
\end{tabular}

a weak band at $\sim 1700 \mathrm{~cm}^{-1}$ is present, typical of a $\mathrm{C}=\mathrm{O}$ stretching vibration, suggesting the presence of a ketone or a carboxylic acid compound. This carbonyl feature has also been observed in the acid residues of IDPs, Orgueil and Murchison and interpreted to be a ketone compound (Flynn et al. 2002). The $\mathrm{C}=\mathrm{O}$ vibration generally involves a large change in dipole moment and is associated with a high intrinsic band strength. Therefore, because the band is weak, the compound carrying this functional group must not be very abundant. The two weak absorptions at 2988 and $2995 \mathrm{~cm}^{-1}$ observed by Flynn et al. (2001) and interpreted as a moderately volatile organic phase are not observed here. This indicates that throughout the carbonate-rich lithology the organic phases can be heterogeneously distributed. We observe small aromatic peaks in the $650-900 \mathrm{~cm}^{-1}$ region, and in the $1400-1650 \mathrm{~cm}^{-1}$ region. The latter, however, can also be attributed to alkenes (unsaturated aliphatics) and thus could represent vinyl derivatives. It could thus be argued that the absence of peaks in the region at $3030 \mathrm{~cm}^{-1}$, where the main aromatic $\mathrm{C}-\mathrm{H}$ stretch band typically appears, makes our identification of aromatics only tentative. Nevertheless, the Raman bands in our analyses clearly show the presence of a polyaromatic carbon structure. In fact, the strength of the aromatic $\mathrm{C}-\mathrm{H}$ stretch is generally intrinsically weaker than the aliphatic $\mathrm{C}-\mathrm{H}$ stretch. In addition, there are more $\mathrm{C}-\mathrm{H}$ bonds per $\mathrm{C}$ atom in aliphatics than in aromatics. Thus, even small amounts of aliphatics can dominate the $\mathrm{C}-\mathrm{H}$ stretching region when mixed with aromatics, making the peak at $3030 \mathrm{~cm}^{-1}$ so small that it will appear below the detection limit of the instrument. This would mean that the quantity of aromatics containing $\mathrm{C}-\mathrm{H}$ groups present in Tagish Lake is less than the quantity usually observed in the other carbonaceous chondrites' kerogens. For example, in the acid residue of Orgueil (see Fig. 2 of Ehrenfreund et al. 1991) one can clearly see a feature at $\sim 3040 \mathrm{~cm}^{-1}$ corresponding to the aromatic C-H stretch. This same feature is observed in the acid residue of Murchison (see Fig. 3 of Ehrenfreund et al. 1991), but at $\sim 3030 \mathrm{~cm}^{-1}$. Thus, Murchison and Orgueil contain more aromatic $\mathrm{C}-\mathrm{H}$ than Tagish Lake, which is another difference that can be found among these carbonaceous chondrites. Given these observations, the lack in Tagish Lake of the $3030 \mathrm{~cm}^{-1}$ feature typically of aromatic $\mathrm{C}-\mathrm{H}$ stretch could be due to a higher degree of interlinking between the aromatic domains, as 

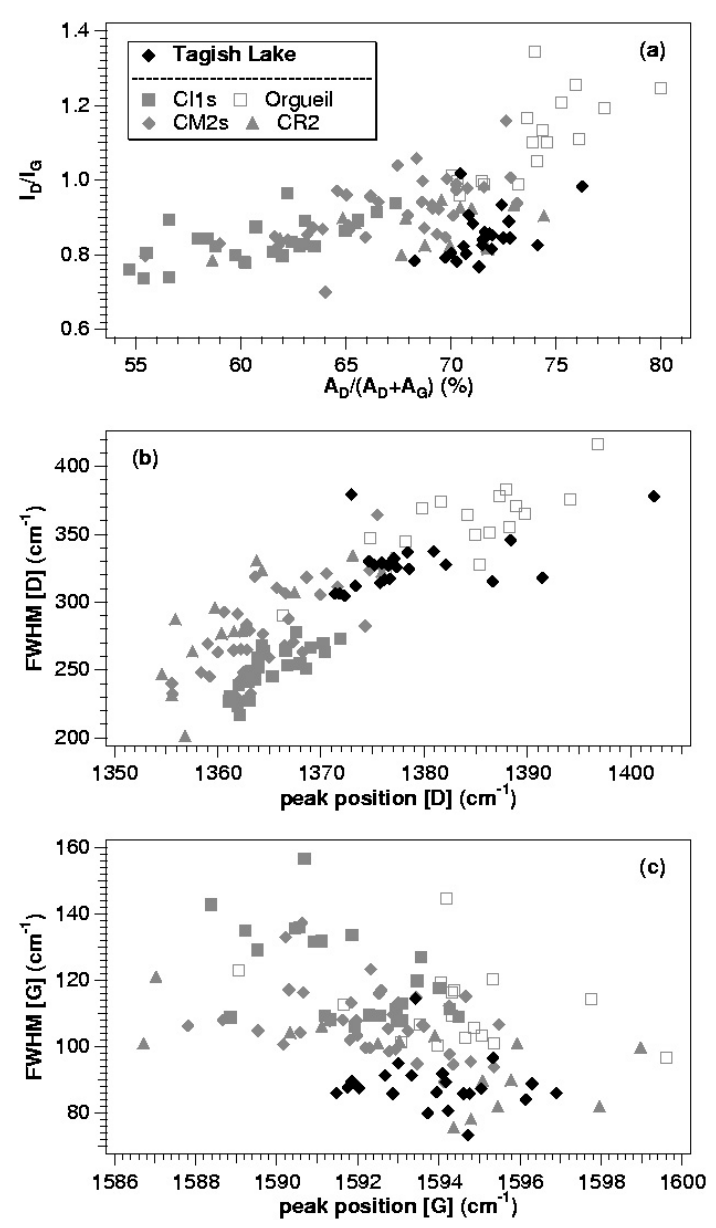

Fig. 6. Comparison of Raman data of Tagish Lake with other carbonaceous chondrites. a) The peak intensities vs. the relative peak areas. b) The width vs. the position: $\mathrm{D}$ band. c) The width vs. the position: G band. Data of carbonaceous chondrites from Raynal (2003).

well as the aliphatic chains. This would result in near loss (or important reduction) of the $3030 \mathrm{~cm}^{-1}$ feature in the IR spectra, but not the $\sim 1600 \mathrm{~cm}^{-1}$ feature in the Raman spectra, which is due to aromatic $\mathrm{C}=\mathrm{C}$ modes and which does not decrease with increased interlinkage. Considering the points described above, the aromatic compound found in the carbonate-rich lithology of Tagish Lake must be only composed of $\mathrm{C}=\mathrm{C}$ groups and no (or below the instrument detection limit) $\mathrm{C}-\mathrm{H}$ groups.

The comparison of our FTIR results with those obtained by Nakamura et al. (2002) on the carbonate-poor lithology shows that the diversity and distribution of the organics present in both lithologies are different: while the carbonaterich lithology studied in this work shows aliphatics, a ketone or a carboxylic group, and a polyaromatic compound mainly composed of $\mathrm{C}=\mathrm{C}$, the carbonate-poor lithology studied by Nakamura et al. (2002) contains aliphatics, esters, and aromatics.

IR analyses were made in the organic material extracted from Orgueil (Ehrenfreund et al. 1991) and Murchison (Ehrenfreund et al. 1991; de Vries et al. 1993). The comparison of the $2700-3000 \mathrm{~cm}^{-1}$ spectral region of these two carbonaceous chondrites with our spectra of Tagish Lake shows that they are quite different. While in both Orgueil and Murchison the methyl group $\left(\mathrm{CH}_{3}\right)$ bands (both symmetric and asymmetric) are prominent related to the methylene group $\left(\mathrm{CH}_{2}\right)$ band (only the band at $\sim 2925 \mathrm{~cm}^{-1}$ is present), in the spectra of Tagish Lake one can see the opposite: the methylene group bands (both symmetric and asymmetric) are prominently related to the methyl group bands (the symmetric feature at $\sim 2865 \mathrm{~cm}^{-1}$ is very weak). These differences support the previous conclusions (Zolensky et al. 2002) that Tagish Lake is indeed a new kind of meteorite.

\subsection{Raman}

Our Raman analyses reveal the presence of carbonaceous structures made of molecules having conjugated bonds in all the Tagish Lake pieces analysed. This is consistent with observations in other primitive carbonaceous chondrites (Cronin et al. 1988; Raynal 2003) and is the signature of the presence of highly disordered carbon in this carbonate-rich lithology. In fact, analyses done with Nuclear Magnetic Resonance (NMR) of the insoluble macromolecular carbon of Tagish Lake revealed that this material is predominantly composed of aromatic carbon, and differs from those of Orgueil, Murchison, and Allende (Cody et al. 2002; Pizzarello et al. 2001).

The plot (Fig. 6) of the Raman parameters obtained for Tagish Lake and other carbonaceous chondrites so far studied (CI1, CM2, CR2 and Orgueil) also shows that the macromolecular carbon of Tagish Lake is different, because although some of the points of Tagish Lake overlap with some of the members of the other meteoritic groups, most of them extend the distribution and tend to separate in the plot from the data of the other carbonaceous chondrites. These results, together with the FTIR results, are consistent with the NMR and GCMS results (Pizzarello et al. 2001) in that Tagish Lake is unique in its organic chemistry.

The Raman spectrum of Tagish Lake can also be compared to the Raman spectra obtained on individual interplanetary dust particles (IDPs). Wopenka (1988) investigated 20 IDPs and classified them according to their Raman spectra in six groups. In Fig. 1 of Wopenka (1988) one can see these groups. In particular, the comparison of the spectra of particles belonging to the group 3 to the spectrum of Tagish Lake shown in Fig. 5 of the present work, shows that they are quite similar, suggesting therefore that Tagish Lake and the IDPs of group 3 have a similar disordered carbon. FTIR analyses of the four IDP particles of group 3 revealed they are composed of layer lattice silicates [LLS] (Sandford \& Walker 1985). Tagish Lake is also mainly composed of LLS. Thus, it would seem that the type of carbonaceous component found in both LLS-IDPs and Tagish Lake is associated with the type of silicate present in the sample. IDPs of group 3 and Tagish Lake have therefore a similar aromatic component.

\subsection{Comparison with astronomical data}

Sandford et al. (1991) demonstrated that the bands of the $\mathrm{C}-\mathrm{H}$ stretching features seen in the diffuse ISM correlated with visual extinction and concluded that the features are due to 
Table 3. FTIR asymmetric parameters obtained for Tagish Lake after a Lorentzian fit. Legend: $\tau=$ absorbance, $\Delta v=$ full-width-at-halfmaximum in $\mathrm{cm}^{-1}$.

\begin{tabular}{cc}
\hline \hline $\mathrm{CH}_{2}$ & $\mathrm{CH}_{3}$ \\
$\left(2922 \mathrm{~cm}^{-1}\right)$ & $\left(2958 \mathrm{~cm}^{-1}\right)$ \\
\hline$\tau=0.96135$ & 0.22014 \\
$\Delta v=25.5$ & 16.8 \\
\hline
\end{tabular}

organic solid materials found in the diffuse ISM. This finding opened the perspective of comparing the IR data of the organics found in carbonaceous meteoritical materials with these astronomical IR features as a way of understanding the formation of organic material in various environments of the Universe. Following this line, Ehrenfreund et al. (1991) compared the IR spectrum of the galactic center source IRS 7 to a spectrum of an organic polymer extracted from Orgueil and found a very good match in the $3.4 \mu \mathrm{m}$ region. Later, Pendleton et al. (1994) investigated the spectral structure of the dust in the diffuse ISM and compared the IR spectra of these objects to that of the carbonaceous component of the Murchison meteorite; there again, they found some similarities between the diffuse ISM and the IR spectrum of Murchison.

To compare our results obtained with the Tagish Lake meteorite to the data known for the diffuse Interstellar Medium (ISM), we have compared the spectra obtained in Tagish Lake to the spectra of the various diffuse ISM objects studied so far, and estimated the $\mathrm{CH}_{2} / \mathrm{CH}_{3}$ abundance ratio in the carbonaceous component of Tagish Lake by approximating the asymmetric bands of the IR spectra using a Lorentzian model. The parameters obtained after this fitting are shown in Table 3.

The comparison of the spectra of objects in the diffuse ISM to the spectra of Tagish Lake in the $3000 \mathrm{~cm}^{-1}$ region shows they are different. In Fig. 7 we show the comparison of the spectrum of the IRS 7 source to the spectrum of Tagish Lake. These two spectra are different in that in the IRS 7 one the $\mathrm{CH}_{3}$ and $\mathrm{CH}_{2}$ features are comparable, whereas the spectra of Tagish Lake is dominated by the $\mathrm{CH}_{2}$ features. Indeed, the diffuse ISM spectra were found to be similar to the Murchison and Orgueil IR spectra (Ehrenfreund et al. 1991; Pendleton et al. 1994) and therefore it is expected that they are different from the spectra of Tagish Lake. This difference is supported by the comparison between the ratio $\mathrm{CH}_{2} / \mathrm{CH}_{3}$ of both Tagish Lake and the diffuse ISM. While for the former we find a value of 7.3, the ratio found in the diffuse ISM is 2.5 (Sandford et al. 1991; Pendleton et al. 1994). This suggests that the carbonaceous component of Tagish Lake is different from the one found in the diffuse ISM, having longer aliphatic chains. This implies that the interlinking within the aliphatics in Tagish Lake is higher than the one found in the diffuse ISM. Because in our spectra aromatics and the carbonyl group $\mathrm{C}=\mathrm{O}$ are present, we can also conclude that these aliphatic chains are associated with electronegative chemical groups containing $\mathrm{C}=\mathrm{O}$ and aromatic moieties, such as has been found in the diffuse ISM (Sandford et al. 1991; Pendleton et al. 1994; Sandford et al. 1995). Furthermore, the comparison of the

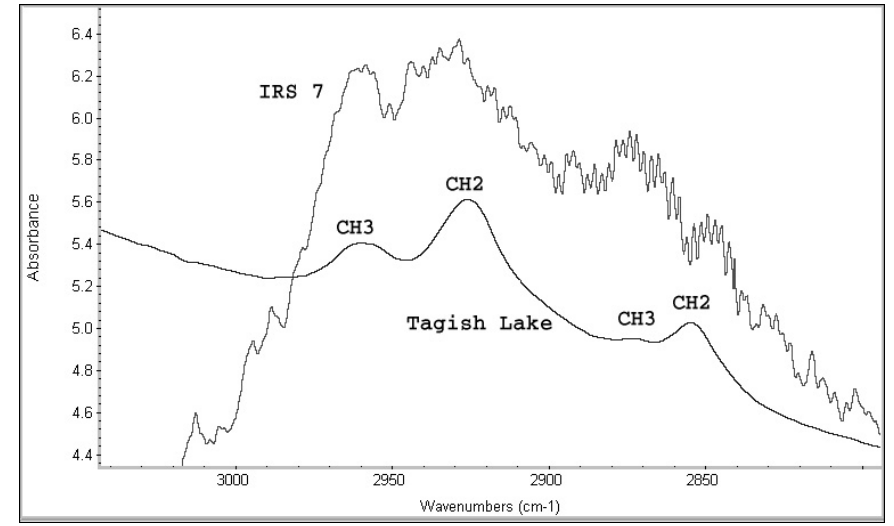

Fig. 7. A comparison of the $3.4 \mu \mathrm{m}$ region $\left(2800-3000 \mathrm{~cm}^{-1}\right)$ of the IRS 7 source to the Tagish Lake meteorite. The IRS 7 source spectrum has been obtained from the Infrared Space Observatory (ISO) Data Center.

IR spectra obtained in the present work to the IR data obtained with irradiation residues (Pendleton et al. 1994) and the organic residue of Murchison and Orgueil (Ehrenfreund et al. 1991; Pendleton et al. 1994) also shows that the Tagish Lake carbonaceous material is different in that it has longer aliphatic chains, thus higher interlinkage within aliphatics than the one found in Orgueil and Murchison. This observation is consistent with previous results obtained with chromatographic techniques, which showed that the organic compounds, in particular the carboxylic acids in Tagish Lake, have a higher ratio of linear to branched acids than the Murchison meteorite (Pizzarello et al. 2001). Tagish Lake is therefore richer in $\mathrm{CH}_{2}$ groups.

\section{Conclusions}

The data obtained during this work show that the carbonaterich lithology of Tagish Lake is mainly composed of phyllosilicates, aliphatic hydrocarbons, and highly disordered polyaromatic carbonaceous material. Compared to previous studies, it is clear that the organic material in Tagish Lake is heterogeneously distributed throughout the meteorite, having a differential distribution of the organics in the two lithologies. In particular, the IR data show that the Tagish Lake meteorite has longer aliphatic chains than Murchison, Orgueil and the diffuse ISM. Moreover, the Raman data show that much of the carbon presented in the matrix of this meteorite is composed of aromatics mainly composed of $\mathrm{C}=\mathrm{C}$ modes.

In general, both FTIR and Raman data show that Tagish Lake has an atypical carbonaceous component when compared to other carbonaceous chondrites, supporting the assertion that this is a new, unique meteorite. However, because the plot of its Raman parameters does not position Tagish Lake between the CI and CM2 groups, it would be interesting to consider more carefully the carbon structure, composition, and diversity for the classification of this meteorite.

The IR data obtained in this work shows that the peaks observed in the $3.4 \mu \mathrm{m}$ region of the diffuse ISM are also found in Tagish Lake; however, the ratio $\mathrm{CH}_{2} / \mathrm{CH}_{3}$ measured in Tagish Lake is different from the one observed in the diffuse ISM and also from the one measured in the carbonaceous 
chondrites Murchison and Orgueil. Therefore, the presence of similar peaks does not necessarily mean that the organic compound present in all these different objects is the same. This suggests that the processes that formed the organics observed in Tagish Lake could be different from the processes that formed the organics observed in other meteorites, IDPs and in the diffuse ISM. Whether this difference means that the processes that formed the organics in Tagish Lake took place in a different environment, and/or through a different pathway, is not clear; a more careful investigation is still needed for a precise correlation to be established.

The FTIR and Raman microspectroscopies constitute a parallel approach to the electron microscopy and the chromatographic/chemical methods for the analysis of rare samples, such as the soon-to-be-collected STARDUST cometary grains.

Acknowledgements. We acknowledge the CNES Exobiologie program who funded GM. We thank P. Dumas and G. Montagnac for their assistance during the FTIR and Raman analyses, respectively and E. Dartois and G. Muñoz Caro for helpful discussion. We are grateful to S. Sandford who provided very constructive comments to this manuscript.

\section{References}

Baker, L., Franchi, I. A., Wright, I. P., \& Pillinger, C. T. 2002, Meteorit. Planet. Sci., 37, 977

Brown, P., Hildebrand, A., Zolensky, M., et al. 2000, Science, 290, 320

Christophe Michel-Lévy, M., \& Lautié, A. 1981, Meteoritics, 16, 301

Cody, G. D., O’D. Alexander, C. M., \& Tera, F. 2002, Lunar Planet. Sci., 33, \# 1806

Cronin, J., Pizzarello, S., \& Cruikshank, D. 1988, in Meteorites and the Early Solar System, ed. M. S. Matthews, \& J. F. Kerridge (Tucson: Univ. of Arizona Press), 819

de Vries, M. S., Reihs, K., Wendt, H. R., et al. 1993, Geochim. Cosmochim. Acta, 57, 933

D’Hendecourt, L. B., \& Allamandola, L. J. 1986, A\&A, 64, 453

Ehrenfreund, P., Robert, F., D’Hendencourt, L., \& Behar, F. 1991, A\&A, 252, 712

Ferrari, A., \& Robertson, J. 2000, Phys. Rev. B, 61, 14095

Flynn, G. J., Keller, L. P., Jacobsen, C., \& Wirick, S. 2001, Lunar Planet. Sci., 32, \# 1593

Flynn, G. J., Keller, L. P., Joswiak, D., \& Brownlee, D. E. 2002, in Lunar and Planetary Institute Conference Abstracts, \# 1320

Friedrich, J. M., Wang, M., \& Lipschutz, M. E. 2002, Meteorit. Planet. Sci., 37, 677
Grady, M. M., Verchovsky, A. B., Franchi, I. A., Wright, I. P., \& Pillinger, C. T. 2001, Meteorit. Planet. Sci., 36, A71

Grady, M. M., Verchovsky, A. B., Franchi, I. A., Wright, I. P., \& Pillinger, C. T. 2002, Meteorit. Planet. Sci., 37, 713

Heymann, D., Burke, E., \& Touret, J. 1986, Lunar Planet. Sci., 339

Hiroi, T., Zolensky, M. E., \& Pieters, C. M. 2001, Science, 293, 2234

Keller, L. P., \& Flynn, G. J. 2001, Lunar Planet. Sci., 32, \# 1639

Kminek, G., Botta, O., Glavin, D. P., \& Bada, J. L. 2002, Meteorit. Planet. Sci., 37, 697

Leshin, L. A., Farquhar, J., Guan, Y., et al. 2001, Lunar Planet. Sci., 32, \# 1843

Macklin, J., Brownlee, D., Chang, S., \& Bunch, T. 1990, in Carbon in the Galaxy: studies from Earth and Space, 336

Makjanic, J., Touret, J. L. R., Vis, R. D., \& Verheul, H. 1989, Meteoritics, 24, 49

Mittlefehldt, D. W. 2002, Meteorit. Planet. Sci., 37, 703

Nakamura, K., Nakashima, S., Shiota, D., Zolensky, M. E., \& Keller, L. P. 2003a, Lunar Planet. Sci., 34, \# 1432

Nakamura, K. T., Zolensky, M. E., Tomita, S., Nakashima, S., \& Tomeoka, K. 2002, Int. J. Astrobiol., 1, 179

Nakamura, T., Noguchi, T., Zolensky, M. E., \& Tanaka, M. 2003b, Earth Planet. Sci. Lett., 207, 83

Pendleton, Y. J., Sandford, S. A., Allamandola, L. J., Tielens, A. G. G. M., \& Sellgren, K. 1994, ApJ, 437, 683

Pizzarello, S., \& Huang, Y. 2002, Meteorit. Planet. Sci., 37, 687

Pizzarello, S., Huang, Y., Becker, L., et al. 2001, Science, 293(5538), 2236

Raynal, P.-I. 2003, Ph.D. Thesis, Université Pierre et Marie Curie, Paris, France

Raynal, P. I., Quirico, E., Borg, J., et al. 2000, Planet. Space Sci., 48, 1329

Rietmeijer, F. J. M. 1990, in Carbon in the Galaxy: studies from Earth and Space, 339

Salisbury, J. W., Walter, L. S., Vergo, N., \& D'Aria, D. M. 1992, Infrared $(2.1-25 \mu \mathrm{m}$ ) spectra of minerals (The Johns Hopkins University)

Sandford, S. A., Allamandola, L. J., Tielens, A. G. G. M., et al. 1991, ApJ, 371, 607

Sandford, S. A., Pendleton, Y. J., \& Allamandola, L. J. 1995, ApJ, 440,697

Sandford, S. A., \& Walker, R. M. 1985, ApJ, 291, 838

Socrates, G. 2001, Infrared and Raman characteristic group frequencies., 3rd ed. (Wiley and Sons, LTD.)

Wopenka, B. 1988, Earth Planet. Sci. Lett., 88, 221

Wopenka, B., \& Sandford, S. A. 1984, Lunar Planet. Sci., 537

Zolensky, M. E., Nakamura, K., Gounelle, M., et al. 2002, Meteorit. Planet. Sci., 37, 737 